\title{
Expanding the treatment window for ischemic stroke through the application of novel system-based technology
}

\author{
Mohammad El-Ghanem, MD, ${ }^{1}$ Fawaz AI-Mufti, MD, ${ }^{1}$ Venkatraman Thulasi, BA, ${ }^{2}$ \\ Inder Paul Singh, MD, MPH, ${ }^{1}$ and Chirag Gandhi, MD' \\ 1Department of Neurosurgery, ${ }^{2}$ Rutgers University, New Jersey Medical School, Newark, New Jersey
}

\begin{abstract}
Recent randomized controlled trials have demonstrated the superiority of endovascular treatment (ET) over medical management in the treatment of acute ischemic stroke patients with anterior circulation emergent large vessel occlusions (ELVOs). Due to such accumulating evidence, expanding ET has become of paramount importance. Advancements in modern technology have enabled the use of mobile stroke units, telestroke networks, mobile neuroendovascular teams, and smartphone applications that shorten the time window to treatment and, thus, make patients more amenable to ET. Additionally, modifying stroke-screening tools to make them more accessible to first responders and the creation of stroke registries can provide further opportunities for ET.
\end{abstract}

https://thejns.org/doi/abs/10.3171/2017.1.FOCUS16515

KEY WORDS acute stroke; telestroke; prehospital stroke scale; endovascular treatment; emergent large vessel occlusion; mechanical thrombectomy; mobile stroke unit; EMS

$\mathrm{B}$ ECAUSE of strict time constraints and various exclusion criteria prohibiting the use of intravenous tissue plasminogen activator (tPA), only $5 \%$ of patients with ischemic stroke in the US and Europe are actually treated with this thrombolytic therapy. ${ }^{1,27}$ Mechanical thrombectomy, however, is effective for up to 6 or more hours following symptom onset. ${ }^{24}$ Recently, multiple randomized controlled trials demonstrated that endovascular treatment (ET) affords patients a $13.5 \%$ higher chance of achieving good clinical outcomes when compared with patients who received intravenous thrombolysis alone. ${ }^{5,32}$

Another major obstacle in stroke treatment is the shortage of cerebrovascular specialists and high-level specialty stroke centers. Despite the occurrence of nearly 800,000 strokes annually in the US, there are only 1100 stroketrained specialists available. ${ }^{25}$ Making matters worse is the fact that many areas with a disproportionately high incidence of stroke, such as the Southeastern US, have the lowest density of cerebrovascular specialists. ${ }^{2,8,11}$

Acknowledgment of the time-critical nature of stroke treatment and the shortage of cerebrovascular specialist has led to many attempts to modify how our health care system approaches stroke care. Many of these pursuits have been spurred by modern advancements in technology and communication systems. Examples include the implementation of telestroke, educating emergency medical services (EMS) personnel in localizing lesions using screening tools, and the advent of mobile stroke units (MSUs), mobile embolectomy teams, and smartphone applications that can be used by both physicians and patients to aid stroke care.

\section{Methods to Expand Stroke Care EMS and Prehospital Stroke Screening}

With mounting evidence of the benefits of ET in the treatment of ischemic stroke due to large vessel occlusion, it is imperative that these patients be promptly transported to an ET facility. In an effort to improve care and reduce the morbidity and mortality caused by stroke, the American Heart Association recommendations include the adoption of EMS protocols for the identification and rapid transport of acute stroke patients to primary stroke centers. ${ }^{17}$ Furthermore, it is recommended that EMS responders preliminarily notify the receiving stroke center to activate the hospital-based acute stroke team of the

ABBREVIATIONS CPSSS = Cincinnati Prehospital Stroke Severity Scale; ELVO = emergent large vessel occulion; EMS = emergency medical services; ET = endovascular treatment; FAST-ED = Field Assessment Stroke Triage for Emergency Destination; KMET = Kanazawa mobile embolectomy team; MSU = mobile stroke unit; NIHSS = National Institutes of Health Stroke Scale; PASS = Prehospital Acute Stroke Severity; RACE = Rapid Arterial Occlusion Evaluation; tPA = tissue plasminogen activator; VAN = Vision, Aphasia, Neglect.

SUBMITTED December 1, 2016. ACCEPTED January 17, 2017.

INCLUDE WHEN CITING DOI: 10.3171/2017.1.FOCUS16515. 
incoming patient, which includes the endovascular team in some centers. ${ }^{13}$ Therefore, stroke care systems are designed to streamline fast recognition, transport, and the initiation of care for acute stroke victims by establishing strategies for preferentially routing stroke patients to designated stroke centers.

In addition, the results of recent ET trials have shown that the recognition of emergent large vessel occlusions (ELVOs) in acute stroke patients is imperative because it necessitates transport to a comprehensive stroke center with ET capabilities.

This process would be greatly facilitated if emergency medical personnel could suspect the presence of large vessel occlusions in stroke patients using a screening tool. Currently, the gold standard for the evaluation of stroke is the National Institutes of Health Stroke Scale (NIHSS). A drawback of this scale is that it often lacks information about stroke severity and the presence or absence of large vessel occlusion. While it is possible to predict the region of the large vessel occlusion using the NIHSS scale, it is often too complicated for emergency medical personnel to execute in the field. If paramedics could derive this information in the field, it would greatly aid neurointerventionalists by expediting the treatment process. For example, if a screening test could help EMS personnel determine the presence of a large vessel occlusion stroke, the patient could be directly transported to an endovascular-capable facility rather than a primary stroke center. ${ }^{28}$ Emergency responders can use several screening scores as an alternative to the NIHSS (see Table 1) as detailed below.

\section{Rapid Arterial Occlusion Evaluation}

The Rapid Arterial Occlusion Evaluation (RACE) scale is scored based on facial palsy (range 0-2), arm motor function (range 0-2), leg motor function (range 0-2), gaze (range $0-1$ ), and aphasia or agnosia (range $0-2$ ). In a 2014 study, RACE was tested in the field by trained emergency medical technicians, and the results were confirmed by neurologists using MR angiography, transcranial duplex sonography, and CT. In the prospective cohort, the results of using the RACE scale in the prehospital setting by emergency medical technicians were comparable to those of NIHSS done by a neurologist in a hospital setting. ${ }^{34}$ The predictive value of this scale was best when the score was $\geq 5$, for which the sensitivity was 0.85 and the specificity was 0.68 . The study supported the notion that the RACE scale is a simpler alternative to NIHSS and can be used by emergency medical technicians to identify large vessel occlusions. There have been numerous attempts to simplify the RACE scale; however, studies showed that the modifications resulted in a lower predictive value than the original.? In the simplified RACE scales, the correct classification of ELVOs was lowered by $9 \%$ if facial palsy was simplified or gaze deviation was removed and lowered by $4.5 \%$ if aphasia or agnosia was removed.

\section{Prehospital Acute Stroke Severity}

The Prehospital Acute Stroke Severity (PASS) scale is based on NIHSS, and the score is based on the level of consciousness, gaze palsy/deviation, and arm weakness. In a 2016 study in Denmark, the PASS scale identified nearly
2 of 3 patients with large vessel occlusion, which was later confirmed by CT or MR angiography. The simplicity of the PASS scale derives from the fact that it contains only 3 clinical elements and the presence of at least 2 of those elements is reason to suspect ELVO with high specificity and a comparable NIHSS score. ${ }^{14}$ The high specificity (median 0.83 ; 95\% CI $0.81-0.85$ ) and predictive value of the PASS scale derives from the fact that gaze abnormalities are often associated with ELVO.

\section{Field Assessment Stroke Triage for Emergency Destination}

The Field Assessment Stroke Triage for Emergency Destination (FAST-ED) scale is based on certain components of NIHSS that have a high predictive value for ELVOs such as facial palsy (range $0-1$ ), arm weakness (range $0-2$ ), speech changes (range $0-2$ ), time (range 0-2), eye deviation (range $0-2$ ), and denial/neglect (range $0-2$ ). The performance of the FAST-ED scale was compared with NIHSS, the RACE scale, and the Cincinnati Prehospital Stroke Severity Scale (CPSSS) in the Screening Technology and Outcomes Project in Stroke cohort. The results, which were confirmed by CT angiography, showed that the FAST-ED scale was as accurate as the more complicated NIHSS for the prediction of large vessel occlusion and was more accurate than the RACE scale and CPSSS. At scores $\geq 4$, the FAST-ED scale had a sensitivity of 0.61 and a specificity of 0.89 . The FAST-ED scale was especially accurate in detecting proximal vessel occlusion, especially those of the internal carotid artery and $\mathbf{M}_{1}$ segment of the middle cerebral artery, which are the most responsive to ET. ${ }^{28}$

\section{Vision, Aphasia, Neglect Screening Tool}

The Vision, Aphasia, Neglect (VAN) Screening Tool tests motor weakness, vision, aphasia, and neglect and is tailored to test multiple cortical divisions of the middle cerebral artery. This screening tool does not have a score that needs to be calculated and can be used by emergency responders or clinicians in the emergency department to assess functional neurovascular anatomy. In a 2016 pilot study, the VAN screening tool was used by triage nurses in 62 patients suspected of stroke prior to vascular imaging. The results showed that of the 19 patients who tested positive on VAN, all were considered for embolectomy (100\% sensitivity) and 5 did not have an emergent large vessel occlusion (90\% specificity). ${ }^{42}$

\section{Cincinnati Prehospital Stroke Severity Scale}

The Cincinnati Prehospital Stroke Severity Scale (CPSSS) is a 3-item stroke scale based on a simplified version of NIHSS. The score ranges from 0 to 4 and is based on the presence of conjugate gaze (range 0-2), arm weakness (range $0-1$ ), and abnormal levels of consciousness (range 0-1). CPSSS was developed to provide a userfriendly prehospital stroke scale that could be accessible to emergency medical technicians and paramedics. This scale was derived based on a study that analyzed which components of NIHSS were most predictive of severe stroke. A 2015 study found that when the CPSSS score was $\geq 2$, sensitivity was $89 \%$ and specificity was $73 \% .20$ 
TABLE 1. Prehospital stroke scales for large vessel occlusion ischemic stroke

\begin{tabular}{|c|c|c|c|c|c|}
\hline $\begin{array}{l}\text { Screening } \\
\text { Method }\end{array}$ & Components & Sensitivity & Specificity & $\begin{array}{c}\text { Positive } \\
\text { Predictive } \\
\text { Value }\end{array}$ & $\begin{array}{c}\text { Negative } \\
\text { Predictive } \\
\text { Value }\end{array}$ \\
\hline NIHSS* & $\begin{array}{l}\text { Consciousness, horizontal eye movement, visual field test, facial palsy, motor of } \\
\text { arm, motor of leg, limb ataxia, sensory, language, speech, extinction \& inattention }\end{array}$ & 0.76 & 0.70 & 0.55 & 0.85 \\
\hline $\mathrm{RACE}^{34} \dagger$ & Facial palsy, arm motor function, leg motor function, gaze, aphasia/agnosia & 0.85 & 0.68 & 0.42 & 0.94 \\
\hline PASS $^{14}$ & Consciousness, gaze palsy/deviation, arm weakness & 0.66 & 0.83 & 0.68 & 0.81 \\
\hline FAST-ED ${ }^{28} \ddagger$ & Facial palsy, arm weakness, speech changes, time, eye deviation, denial/neglect & 0.61 & 0.89 & 0.72 & 0.82 \\
\hline $\mathrm{VAN}^{42}$ & Motor weakness, vision, aphasia, neglect & 1.00 & 0.90 & 0.74 & 1.00 \\
\hline CPSSS $^{20}$ & Conjugate gaze, arm weakness, abnormal levels of consciousness & 0.89 & 0.73 & 0.89 & 0.62 \\
\hline
\end{tabular}

* All included components for a score $\geq 6$.

$\dagger$ All included components for a score $\geq 5$.

$\ddagger$ All included components for a score $\geq 4$.

\section{Telestroke}

Telestroke is an expansion of the concept of telemedicine, which originated in the $1950 \mathrm{~s}^{39}$ with the aim of providing remote care to patients using a communication network. The concept of "telestroke" was first introduced in 1999 by Levine and Gorman with the aim of providing acute stroke care to patients in areas devoid of stroke specialty centers, usually patients in rural settings. ${ }^{26}$ Telestroke is typically applied through a hub and spoke model in which smaller spoke hospitals (without stroke specialty care) are connected to a major hub hospital (with stroke specialty care). When the spoke hospitals get stroke patients, they engage in consultation with a stroke specialist at the hub hospital via a telestroke network. A cost effectiveness study of telestroke using the hub and spoke model found that when compared with having no network, a single hub with 7 spoke hospitals resulted in 45 more patients being treated with intravenous tPA, 20 more patients being treated with endovascular stroke therapies, and 5 additional patients recovering to independence per 1000 acute ischemic strokes per year. ${ }^{40}$

Telestroke plays an active role in expanding endovascular outreach. For example, the determination of large vessel occlusions using screening tools via video conferencing enables providers to select candidates for ET. In addition, sending vascular images remotely allows central providers to determine the candidacy of a patient for ET based on the level of occlusion. A study by the Rush Telestroke Program found that patients who were transferred to the hospital via the telestroke network were more likely to undergo intraarterial treatment (66\% vs 55\%) than those transferred by phone consultation. ${ }^{33}$ The availability of telestroke networks can also facilitate prompt endovascular intervention by allowing the preparation of the facility and tools while the patient is still in transit. ${ }^{3}$ Thus, the implementation of telestroke networks can greatly decrease the burden of stroke by increasing the rates of tPA administration and ET.

One of the drawbacks of telestroke is the additional time required to evaluate the patient because the consulting neurologist will often repeat the entire neurological examination that the emergency department physician probably performed. This further delays an already timesensitive treatment process. In addition, the high startup cost of the equipment and complicated licensure processes have prevented many health care institutions from adopting telestroke.

\section{Mobile Stroke Units}

Despite the implementation of telestroke and other efforts to streamline acute stroke care, the time between symptom onset and the treatment of stroke has plateaued around an average of 130 minutes. ${ }^{4,10,15,16,29,35,37,41,44}$ The greatest delay in the treatment of stroke patients has been shown to be in the prehospital phase, in which the patient is transported to a stroke specialty facility. ${ }^{21}$ In an effort to combat this delay, the concept of the MSU was developed. The aim with the MSU aim is to deliver a comprehensive stroke team with the equipment needed to diagnose and treat a stroke at the scene of the patient.

MSUs are essentially modified ambulances equipped with a CT scanner, CT technologist, nurse, paramedic, and point-of-care laboratory. In addition, some MSUs have an neurologist on board the ambulance, and in others a neurologist at a remote location is available via teleconferencing.

The concept of the MSU was first implemented in Berlin, Germany, by the Stroke Emergency Mobile project, which showed a dramatic shortening in the time to diagnosis and treatment of stroke patients. The Prehospital Acute Neurological Treatment and Optimization of Medical Care in Stroke Study showed that the time from notification to treatment was reduced by 25 minutes and the rate of thrombolysis increased by $12 \%$ in patients treated by an MSU when compared with standard EMS care. ${ }^{10}$ The study also did not report any increases in adverse effects due to tPA administration when compared with traditional treatment. Another controlled study in Saarland, Germany, showed that the use of MSUs allowed a reduction of 41 minutes in the median time needed to make a therapeutic decision from alarm to therapy (35 minutes with MSU vs 76 minutes with regular care). ${ }^{44}$ In addition, in patients given tPA, the time to treatment was nearly halved with a median of 72 minutes.

A recent study showed that an MSU's CT angiography capabilities could allow endovascular candidates to be identified in the field, thus enabling prompt transfer 
to comprehensive stroke centers. As a result, the study showed that the "door-to-groin puncture" time for patients with an ELVO when triaged by an MSU was 103 minutes shorter than the time of EMS dispatch (93 minutes vs 200 minutes).$^{18}$ Theoretically, the reduction in time to treatment enabled by the implementation of MSUs should translate to better clinical outcomes, but studies have shown that patient survival and disability at 3 months after the cerebrovascular incident remain unchanged. ${ }^{22}$

Despite several promising pilot studies, the concept of the MSU has yet to be widely adopted due to the high startup and maintenance costs (of both the equipment and the core MSU team that consists of a paramedic and a stroke physician), radiation safety protocols, special licensure, and lack of data on the cost effectiveness of this approach.

\section{Mobile Neuroendovascular Team}

To address the shortage of ET-trained personnel in certain areas, the concept of a mobile endovascular team was developed. This essentially consists of an endovascular team that travels to remote hospitals to perform ET. This concept was tested in Kanazawa, Japan, by the formation of the Kanazawa mobile embolectomy team (KMET). Basically, on the arrival of a stroke patient to a primary stroke center, the physician of first contact would initiate tPA if indicated and call KMET if the patient met the criteria for endovascular intervention. Once the members of KMET arrived, they would perform ET. The study showed a revascularization rate of $80 \%$, which is similar to that of the recent randomized controlled trials. This enforced the concept that a mobile embolectomy team can be safely applied in real-world conditions. In addition, the time from symptom onset to groin puncture was a mean of 224 minutes despite the mobile embolectomy team having to travel 60 to 70 minutes. On the other hand, randomized controlled trials that have examined mechanical thrombectomy performed in hospital-based neuroangiography suites reported a mean time of symptom onset to groin puncture ranging from 200 to 269 minutes. This confirmed that ET performed by a mobile embolectomy team does not increase the time to revascularization therapy when compared with traditional embolectomies. ${ }^{43}$

The potential drawbacks of treatment by a mobile neuroendovascular team could be an increased rate of technical errors due to operation in an unfamiliar environment. In addition, even minor complications that occur during the recanalization procedure could become highly detrimental due to the lack of personnel expertise and equipment in remote hospitals. Studies have been limited because the concept of mobile neuroendovascular teams is relatively novel, and thus a definitive conclusion about their efficiency cannot be drawn.

\section{Smart Device Applications}

Due to advances in technology and communication, several smartphone applications have been developed that allow physicians and patients to participate in health care at their convenience. A teleradiology application called ResolutionMD serves to send the CT brain scans of patients to the smartphones of vascular neurologists in a telestroke network. This allows the neurologist to assess images at his/her convenience on his/her own mobile device and expedites diagnostic and treatment decisions. A study conducted to compare the interpretation of CT images by neurologists using ResolutionMD and radiologists using the picture archiving and communication system showed that both interpretations were in outstanding agreement with each other. ${ }^{9}$

Stroke119 is a smartphone application that provides a stroke-screening tool based on CPSSS that helps patients recognize the early symptoms of a stroke. Users choose from a set of cartoon images that best mimic the patient's symptoms, and the application predicts the patient's likelihood of undergoing a stroke. In addition, the application provides information about nearby hospitals that offer thrombolytic therapy using the smartphone's global positioning system. ${ }^{31}$ This application helps caregivers and patients determine the possibility of a stroke and navigate to nearby stroke specialty facilities. Twiage (www. twiagemed.com) is another smartphone application developed to allow real-time communication between emergency responders and hospitals while maintaining HIPAA compliance. It enables paramedics to send real-time photos and videos to hospitals to prepare for proper intervention upon the patient's arrival. For stroke patients, the application can be used to send videos of the patient's stroke symptoms to vascular neurologists or telestroke networks.

Smartphone applications can also be used to facilitate medical research and analysis. To collect and analyze information about prehospital stroke care, the smartphoneassisted prehospital medical information system was developed. During the analysis of medical emergencies that lead to hospital admission, information about the prehospital phase is often difficult to find. With this system, information about the prehospital/emergency phase of a patient's care can be quickly searched in combination with his/her in-hospital medical information. ${ }^{30}$ This allows for convenient analysis of the associations between the prehospital and in-hospital phases of various diseases. In addition, because the prehospital component of stroke is of paramount importance, this application can lead to the discovery of novel information about stroke care.

\section{Stroke Registries and Image Analysis Systems}

Recent technological advancements have also facilitated large-scale analyses of the clinical courses of cerebrovascular disorders. Historically, the large amount of clinical and imaging data generated from stroke treatment was contained in disparate medical records. However, the recent creation of online registries to track cerebrovascular disorders allows the use of big data in analyzing stroke treatment. This promotes the use of precision medicine for the treatment of cerebrovascular disorders. Specifically, image-intensive interventional registries enable the detection of patterns in the neuropathophysiology of acute stroke and its response to various therapies. For example, recent randomized controlled trials that extolled the benefits of ET depended on the identification of a favorable collateral circulation profile that was conducive to treatment with ET. ${ }^{38}$

Using this information, optimal treatment methods can 
be established for individual cases. In addition, registries will benefit from the results of recent randomized controlled trials on ET, which have urged the use of imaging and angiography data in determining the candidacy of patients for ET and the use of serial imaging to investigate ischemic tissue in the subacute period. 5,6,12,19,36

In an attempt to automate the analysis of scanned images, RAPID - a system for processing of perfusion- and diffusion-weighted images-was created. This system can automatically detect lesions on perfusion- and diffusion-weighted images within 10 minutes of the scan. ${ }^{23}$ RAPID was mainly created to overcome operator dependence on past MRI-processing systems. The analysis of scanned images is crucial for selecting subsets of ischemic stroke patients who would most likely benefit from reperfusion therapy. A 2012 study showed that the use of RAPID could effectively identify patients in whom reperfusion is associated with an increased chance of a good outcome. ${ }^{23}$

\section{Impact of Endovascular Expansion on Current Stroke Systems}

As emphasized throughout our discussion, the importance of prompt stroke treatment cannot be overstated.
Modifying existing prehospital stroke scales to make them more accessible to emergency responders can enable the localization of lesions and detection of large vessel occlusions, thereby ensuring quicker transport times to the appropriate stroke care facilities. Implementing telestroke networks can increase efficiency and shorten the time to treatment, especially in rural areas devoid of stroke specialty centers. MSUs greatly decrease the time of the prehospital phase of stoke care and would be particularly advantageous in highly trafficked urban areas. Similarly, the concept of mobile endovascular teams is favorable in areas with a shortage of cerebrovascular specialists. The advent of smartphone applications can improve stroke care at a myriad of phases, from prehospital care to research. Refer to Fig. 1 for a summary.

\section{Conclusions}

The use of intravenous tPA and ET has greatly reduced the scourge of stroke. However, the time-critical nature of these therapies has prevented the majority of stroke patients from experiencing their healing properties. Studies on telestroke networks, MSUs, educating EMS personnel in localizing lesions using screening scales, mobile

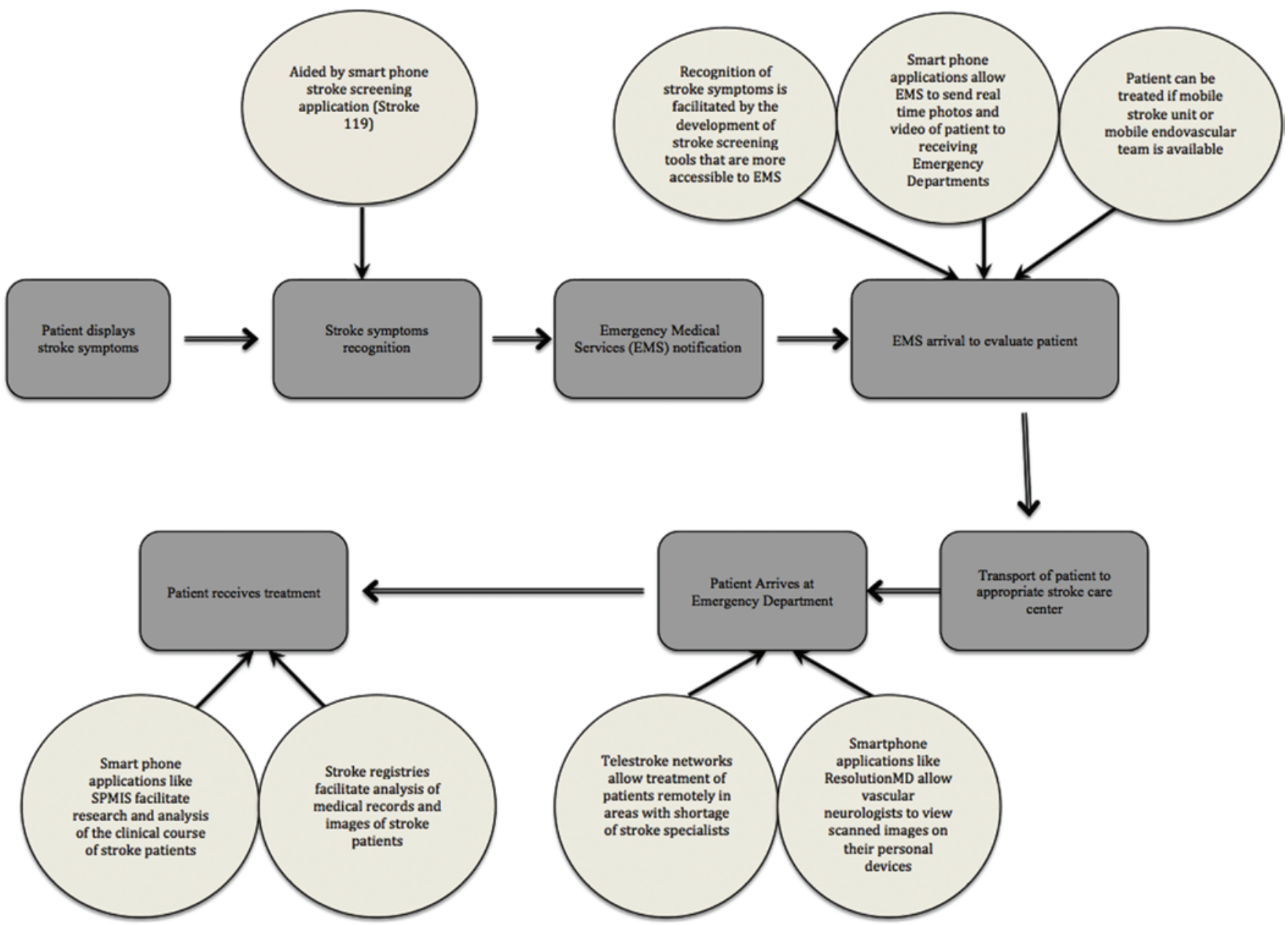

FIG. 1. Application of neuroendovascular outreach interventions into routine acute stroke workflow. SPMIS = smartphone-assisted prehospital medical information system. 
embolectomy teams, and smartphone applications have shown promising results in shortening the time to treatment. These modifications to traditional stroke treatment can translate to improved clinical outcomes.

\section{References}

1. Adeoye O, Hornung R, Khatri P, Kleindorfer D: Recombinant tissue-type plasminogen activator use for ischemic stroke in the United States: a doubling of treatment rates over the course of 5 years. Stroke 42:1952-1955, 2011

2. Adornato BT, Drogan O, Thoresen P, Coleman M, Henderson VW, Henry KA, et al: The practice of neurology, 2000-2010: report of the AAN Member Research Subcommittee. Neurology 77:1921-1928, 2011

3. Akbik F, Hirsch JA, Chandra RV, Frei D, Patel AB, Rabinov JD, et al: Telestroke - the promise and the challenge. Part one: growth and current practice. J Neurointerv [epub head of print], 2016

4. Audebert HJ, Kukla C, Vatankhah B, Gotzler B, Schenkel J, Hofer S, et al: Comparison of tissue plasminogen activator administration management between Telestroke Network hospitals and academic stroke centers: the Telemedical Pilot Project for Integrative Stroke Care in Bavaria/Germany. Stroke 37:1822-1827, 2006

5. Berkhemer OA, Fransen PS, Beumer D, van den Berg LA, Lingsma HF, Yoo AJ, et al: A randomized trial of intraarterial treatment for acute ischemic stroke. N Engl J Med 372:11-20, 2015

6. Campbell BC, Mitchell PJ, Kleinig TJ, Dewey HM, Churilov L, Yassi N, et al: Endovascular therapy for ischemic stroke with perfusion-imaging selection. N Engl J Med 372:10091018,2015

7. Carrera D, Campbell BC, Cortés J, Gorchs M, Querol M, Jiménez X, et al: Predictive value of modifications of the prehospital rapid arterial occlusion evaluation scale for large vessel occlusion in patients with acute stroke. J Stroke Cerebrovasc Dis 26:74-77, 2017

8. Dall TM, Storm MV, Chakrabarti R, Drogan O, Keran CM, Donofrio PD, et al: Supply and demand analysis of the current and future US neurology workforce. Neurology 81:470478, 2013

9. Demaerschalk BM, Vargas JE, Channer DD, Noble BN, Kiernan TE, Gleason EA, et al: Smartphone teleradiology application is successfully incorporated into a telestroke network environment. Stroke 43:3098-3101, 2012

10. Ebinger M, Winter B, Wendt M, Weber JE, Waldschmidt C, Rozanski M, et al: Effect of the use of ambulance-based thrombolysis on time to thrombolysis in acute ischemic stroke: a randomized clinical trial. JAMA 311:1622-1631, 2014

11. Gillum RF, Ingram DD: Relation between residence in the southeast region of the United States and stroke incidence. The NHANES I Epidemiologic Followup Study. Am J Epidemiol 144:665-673, 1996

12. Goyal M, Demchuk AM, Menon BK, Eesa M, Rempel JL, Thornton J, et al: Randomized assessment of rapid endovascular treatment of ischemic stroke. N Engl J Med 372:10191030,2015

13. Hanks N, Wen G, He S, Song S, Saver JL, Cen S, et al: Expansion of U.S. emergency medical service routing for stroke care: 2000-2010. West J Emerg Med 15:499-503, 2014

14. Hastrup S, Damgaard D, Johnsen SP, Andersen G: Prehospital acute stroke severity scale to predict large artery occlusion: design and comparison with other scales. Stroke 47:1772-1776, 2016

15. Hess DC, Wang S, Hamilton W, Lee S, Pardue C, Waller JL, et al: REACH: clinical feasibility of a rural telestroke network. Stroke 36:2018-2020, 2005
16. Hill MD, Buchan AM: Thrombolysis for acute ischemic stroke: results of the Canadian Alteplase for Stroke Effectiveness Study. CMAJ 172:1307-1312, 2005

17. Jauch EC, Saver JL, Adams HP Jr, Bruno A, Connors JJ, Demaerschalk BM, et al: Guidelines for the early management of patients with acute ischemic stroke: a guideline for healthcare professionals from the American Heart Association/ American Stroke Association. Stroke 44:870-947, 2013

18. John S, Stock S, Masaryk T, Bauer A, Cerejo R, Uchino K, et al: Performance of CT angiography on a mobile stroke treatment unit: implications for triage. J Neuroimaging 26:391394, 2016

19. Jovin TG, Chamorro A, Cobo E, de Miquel MA, Molina CA, Rovira A, et al: Thrombectomy within 8 hours after symptom onset in ischemic stroke. N Engl J Med 372:2296-2306, 2015

20. Katz BS, McMullan JT, Sucharew H, Adeoye O, Broderick JP: Design and validation of a prehospital scale to predict stroke severity: Cincinnati Prehospital Stroke Severity Scale. Stroke 46:1508-1512, 2015

21. Köhrmann M, Schellinger PD, Breuer L, Dohrn M, Kuramatsu JB, Blinzler C, et al: Avoiding in hospital delays and eliminating the three-hour effect in thrombolysis for stroke. Int J Stroke 6:493-497, 2011

22. Kunz A, Ebinger M, Geisler F, Rozanski M, Waldschmidt C, Weber JE, et al: Functional outcomes of pre-hospital thrombolysis in a mobile stroke treatment unit compared with conventional care: an observational registry study. Lancet Neurol 15:1035-1043, 2016

23. Lansberg MG, Lee J, Christensen S, Straka M, De Silva DA, Mlynash M, et al: RAPID automated patient selection for reperfusion therapy: a pooled analysis of the Echoplanar Imaging Thrombolytic Evaluation Trial (EPITHET) and the Diffusion and Perfusion Imaging Evaluation for Understanding Stroke Evolution (DEFUSE) Study. Stroke 42:1608-1614, 2011

24. Lee KY, Han SW, Kim SH, Nam HS, Ahn SW, Kim DJ, et al: Early recanalization after intravenous administration of recombinant tissue plasminogen activator as assessed by preand post-thrombolytic angiography in acute ischemic stroke patients. Stroke 38:192-193, 2007

25. Leira EC, Kaskie B, Froehler MT, Adams HP Jr: The growing shortage of vascular neurologists in the era of health reform: planning is brain! Stroke 44:822-827, 2013

26. Levine SR, Gorman M: "Telestroke": the application of telemedicine for stroke. Stroke 30:464-469, 1999

27. Leys D, Ringelstein EB, Kaste M, Hacke W: Facilities available in European hospitals treating stroke patients. Stroke 38:2985-2991, 2007

28. Lima FO, Silva GS, Furie KL, Frankel MR, Lev MH, Camargo EC, et al: Field assessment stroke triage for emergency destination: a simple and accurate prehospital scale to detect large vessel occlusion strokes. Stroke 47:1997-2002, 2016

29. Marler JR, Welch KMA, Tilley BC, Brott T, Lyden P, Grotta $\mathrm{JC}$, et al: Tissue plasminogen activator for acute ischemic stroke. N Engl J Med 333:1581-1587, 1995

30. Nakae T, Kataoka H, Kuwata S, Iihara K: Smartphone-assisted prehospital medical information system for analyzing data on prehospital stroke care. Stroke 45:1501-1504, 2014

31. Nam HS, Heo J, Kim J, Kim YD, Song TJ, Park E, et al: Development of smartphone application that aids stroke screening and identifying nearby acute stroke care hospitals. Yonsei Med J 55:25-29, 2014

32. Nogueira RG, Schwamm LH, Hirsch JA: Endovascular approaches to acute stroke, part 1: drugs, devices, and data. AJNR Am J Neuroradiol 30:649-661, 2009

33. Osteraas N, Conners J, Cutting S, Song S, Cherian L, Diebolt E, et al: Telestroke vs phone consultation in stroke patients 
eligible for intra-arterial therapy (P6.033). Neurology 47 (Suppl 1):ATP292, 2016 (Abstract)

34. Pérez de la Ossa N, Carrera D, Gorchs M, Querol M, Millán M, Gomis M, et al: Design and validation of a prehospital stroke scale to predict large arterial occlusion: the rapid arterial occlusion evaluation scale. Stroke 45:87-91, 2014

35. Sairanen T, Soinila S, Nikkanen M, Rantanen K, Mustanoja S, Färkkilä M, et al: Two years of Finnish Telestroke: thrombolysis at spokes equal to that at the hub. Neurology 76:1145-1152, 2011

36. Saver JL, Goyal M, Bonafe A, Diener HC, Levy EI, Pereira VM, et al: Stent-retriever thrombectomy after intravenous t-PA vs. t-PA alone in stroke. N Engl J Med 372:2285-2295, 2015

37. Schwab S, Vatankhah B, Kukla C, Hauchwitz M, Bogdahn U, Fürst A, et al: Long-term outcome after thrombolysis in telemedical stroke care. Neurology 69:898-903, 2007

38. Sheth SA, Liebeskind DS: Collaterals in endovascular therapy for stroke. Curr Opin Neurol 28:10-15, 2015

39. Shore JH: Telepsychiatry: videoconferencing in the delivery of psychiatric care. Am J Psychiatry 170:256-262, 2013

40. Switzer JA, Demaerschalk BM: Overcoming challenges to sustain a telestroke network. J Stroke Cerebrovasc Dis 21:535-540, 2012

41. Switzer JA, Levine SR, Hess DC: Telestroke 10 years later'telestroke 2.0'. Cerebrovasc Dis 28:323-330, 2009

42. Teleb MS, Ver Hage A, Carter J, Jayaraman MV, McTaggart RA: Stroke vision, aphasia, neglect (VAN) assessment-a novel emergent large vessel occlusion screening tool: pilot study and comparison with current clinical severity indices. J Neurointerv Surg 9:122-126, 2017

43. Uchiyama N, Misaki K, Mohri M, Kamide T, Hirota Y, Higashi $\mathrm{R}$, et al: Treatment result in the initial stage of Kanazawa mobile embolectomy team for acute ischemic stroke. Neurol Med Chir (Tokyo) 56:737-744, 2016

44. Walter S, Kostopoulos P, Haass A, Keller I, Lesmeister M, Schlechtriemen T, et al: Diagnosis and treatment of patients with stroke in a mobile stroke unit versus in hospital: a randomised controlled trial. Lancet Neurol 11:397-404, 2012

\section{Disclosures}

The authors report no conflict of interest concerning the materials or methods used in this study or the findings specified in this paper.

\section{Author Contributions}

Conception and design: El-Ghanem, Al-Mufti, Singh, Gandhi. Drafting the article: El-Ghanem, Al-Mufti, Thulasi. Critically revising the article: El-Ghanem, Al-Mufti, Gandhi. Reviewed submitted version of manuscript: Al-Mufti, Gandhi.

\section{Correspondence}

Mohammad El-Ghanem, Department of Neurosurgery, Rutgers University/New Jersey Medical School, 90 Bergen St., Ste. 8100, Newark, NJ 07103. email: elghanmh@njms.rutgers.edu. 